\title{
The Comparison of Water Absorption Analysis between Counterrotating and Corotating Twin-Screw Extruders with Different Antioxidants Content in Wood Plastic Composites
}

\author{
Mohd Hafizuddin Ab Ghani and Sahrim Ahmad \\ Material Science Programme, School of Applied Physics, Faculty of Science and Technology, National University of Malaysia, \\ 43600 Bangi, Malaysia \\ Correspondence should be addressed to Sahrim Ahmad, sahrim@ukm.my
}

Received 14 February 2011; Revised 11 May 2011; Accepted 31 May 2011

Academic Editor: Krishnan Jayaraman

Copyright ( $) 2011$ M. H. Ab Ghani and S. Ahmad. This is an open access article distributed under the Creative Commons Attribution License, which permits unrestricted use, distribution, and reproduction in any medium, provided the original work is properly cited.

\begin{abstract}
Water absorption is a major concern for natural fibers as reinforcement in wood plastic composites (WPCs). This paper presents a study on the comparison analysis of water absorption between two types of twin-screw extruders, namely, counterrotating and corotating with presence of variable antioxidants content. Composites of mixed fibres between rice husk and saw dust with recycled high-density polyethylene (rHDPE) were prepared with two different extruder machines, namely, counterrotating and corotating twin screw, respectively. The contents of matrix $(30 \mathrm{wt} \%)$ and fibres $(62 \mathrm{wt} \%)$ were mixed with additives $(8 \mathrm{wt} \%)$ and compounded using compounder before extruded using both of the machines. Samples were immersed in distilled water according to ASTM D 570-98. From the study, results indicated a significant difference among samples extruded by counterrotating and corotating twinscrew extruders. The counterrotating twin-screw extruder gives the smallest value of water absorption compared to corotating twin-screw extruder. This indicates that the types of screw play an important role in water uptake by improving the adhesion between natural fillers and the polymer matrix.
\end{abstract}

\section{Introduction}

One of the advantageous features of composite materials is a relatively low water absorption compared to the wood. That is one of the few properties due to which composites are clearly better than virgin wood. This comparison is directly related to dimensional stability and a better durability of the material. Water absorption mainly occurs at the outer layers of composite materials and progressively decreases into the bulk of the matrix. A relatively high water absorption by composite materials leads to a higher weight of wet profiles, possible decrease in their strength, and increase in their deflection, swelling and a resulting pressure on neighboring structures. These can result in buckling, warping, higher chance of their microbial inhabitation, freeze- and thawinduced deterioration of mechanical properties of materials [1].

As discussed by Wang et al. [2], natural fillers such as rice husk and saw dust have a number of advantages over synthetic fillers, since they are abundant resources, environmental friendly, and cheap. They are among a wide variety of lignocellulosic materials that had been used to reinforce thermoplastics. Natural fillers and polymer exhibit discrepancies properties of water uptake; distribution of fillers in polymer is a factor to the overall moisture absorption in composites.

Despite the advantages above, there is a problem associated with using lignocellulosic materials in natural fiber thermoplastic composites which is moisture absorption, biodeterioration, and thickness swell as discussed elsewhere [3, 4]. Due to exposure to the atmosphere, an evaluation of the water resistance is crucial. Hygroscopicity is an undesirable property on natural fibers caused by chemical constituents. The hygroscopicity is one of the factors that need to be addressed to get a full understanding of the mechanism of moisture absorption. The moisture absorption by composites containing natural fibers can affect their long-term performance. 
TABLE 1: Formulation of natural filler rHDPE composites.

\begin{tabular}{lcccc}
\hline \multirow{2}{*}{ rHDPE (wt \%) } & \multicolumn{2}{c}{ Additives (wt \%) } & \multicolumn{2}{c}{ Fillers (wt \%) } \\
& Antioxidants & Other & Rice husk & Saw dust \\
\hline 30 & 0.0 & 8 & 45 & 17 \\
30 & 0.1 & 7.9 & 45 & 17 \\
30 & 0.3 & 7.7 & 45 & 17 \\
30 & 0.5 & 7.5 & 45 & 17 \\
\hline
\end{tabular}

As discussed by Joseph et al. [5], water absorption of composites reinforced with natural fiber can be reduced by improving fiber-matrix adhesion. In order to improve fibermatrix adhesion, coupling agent such as maleic anhydride polyethylene can be used. Stark [6] in her study reported that increased moisture decreased their mechanical properties.

Wood plastic composites (WPCs) are porous. The pores are formed by steam and by volatile organic compounds (VOCs) during extrusion process. Presence of oxygen that flows into pores in WPC which is directly related to the decrease of density (specific gravity) of materials will attack "from inside," particularly at elevated temperatures. The attacks will cause oxidative degradation of WPC. This degradation is the most common reason for a premature mechanical failure in WPC. Oxidative degradation as a result of thermooxidation and photooxidation leads to crumbling, and surface becomes loose, powdery, and weak. In order to prevent oxidative degradation, WPCs commonly contain added antioxidants (AOs), typically in amounts between 0.05 and $0.15 \%$ by weight, and $0.2-0.5 \%$ for stabilization of regrinds. It significantly extends the lifetime of WPC compared to unprotected ones as discussed by Klyosov [1].

\section{Methodology}

Four samples were prepared according to the formulation in Table 1. The matrix is recycled high-density polyethylene (rHDPE). The fillers are mixed between rice husk (60 mesh) and saw dust (40 mesh) to create a hybrid filler. Sawdust is essentially the same thing as wood flour except it is not ground but formed as a byproduct of wood sawing. The rice husk can act as reinforcing fillers at certain optimum level of loading [7]. 2\% of MAPE was added as an additive to improve the compatibility of hydrophobic polymer and hydrophilic rice hulls and thus improves the interfacial bonding between matrix and fillers as discussed by Zabihzadeh [8]. The antioxidants (IRGANOX 1010 and IRGAFOS 168 as a ratio $1: 1$ from Ciba) had been varied to study their presence on water absorption of WPC with different types of extrusion processing machine.

Recycled HDPE, additives, and fillers were compounded in a high-intensity mixer (PRISM PILOT 5 High Speed Mixer) for 20 minutes to produce homogeneous blend. High-intensity mixer is used to premix all materials prior to extrusion. Enough heat is generated to soften the recycled HDPE particle, allowing the thermal stabilizers to adhere to the polymers and providing intimate contact during processing. Then, the mixture was extruded in a laboratory scale counterrotating twin-screw extruder (PolyLab OS RheoDrive 4) for better homogenization of the composite. Gravimetric feeding is used to meter materials into the extruder. The gravimetric feeding is preferred because of adequate feeding regardless of material shape or bulk density, improved product quality control, and material savings can be realized [9]. Barrel temperatures of the four zones were set as $165^{\circ} \mathrm{C}, 170^{\circ} \mathrm{C}, 175^{\circ} \mathrm{C}$, and $180^{\circ} \mathrm{C}$ from feeding to die zones. Screw speed was $80 \mathrm{rpm}$ to reduce the fibres breaking. The extrudates were collected, cooled outside the chamber at room temperature, and granulated into pellets.

WPC test specimens were produced using hot and cold press processes (LP50, LABTECH Engineering Company LTD). Before hot and cold press processes, WPC pellets were ground using grinder to make them fine to suit with the mold. The ground pellets were then put in the mold size $14 \mathrm{~mm} \times 14 \mathrm{~mm} \times 3 \mathrm{~mm}$. The temperatures of the hot press were set $140^{\circ} \mathrm{C}$ for upper platen and lower platen, respectively. The period of preheating, venting, and full pressing were set 3,2,9 minutes, respectively. The cold press was set at 5 minutes to cold the specimens. The pressure used to press the specimens was set at $1000 \mathrm{psi}$ for both hot and cool press. After finishing the molding processes, the specimens had dried in oven for 24 hours with the temperature $70^{\circ} \mathrm{C}$ in order to remove any moisture left. The specimens were then cut according to ASTM specifications for water absorption testing. Water absorption was determined by the weight gain relative to the dry weight of the samples. The same process had been repeated by changing the counterrotating twin-screw with corotating twin-screw extruder (Thermo Prism TSE 16PC).

\section{Flow Chart}

See Figure 1.

\section{Water Absorption Test}

Water absorption study was performed following the ASTM D 570-98 method. Water absorption of the composites was determined after $2 \mathrm{~h}, 12 \mathrm{~h}, 24 \mathrm{~h}$ until 2-month immersion in distilled water at room temperature, $24^{\circ} \mathrm{C}$. Five specimens of each formulation were dried in an oven for $24 \mathrm{~h}$ at $80 \pm$ $2^{\circ} \mathrm{C}$. The dried specimens were weighed with a precision of $0.001 \mathrm{~g}$. All specimens were immersed in distilled water. At the end of the immersion periods, the specimens were removed from the distilled water, the surface water was wiped off using tissue, and wet weight values were determined. Water absorption percent was calculated using the following formula:

$$
M(\%)=\left(m_{t}-m_{o}\right) / m_{o} \times 100,
$$

where $m_{o}$ and $m_{t}$ denote the oven-dry weight and weight after time $t$, respectively. 


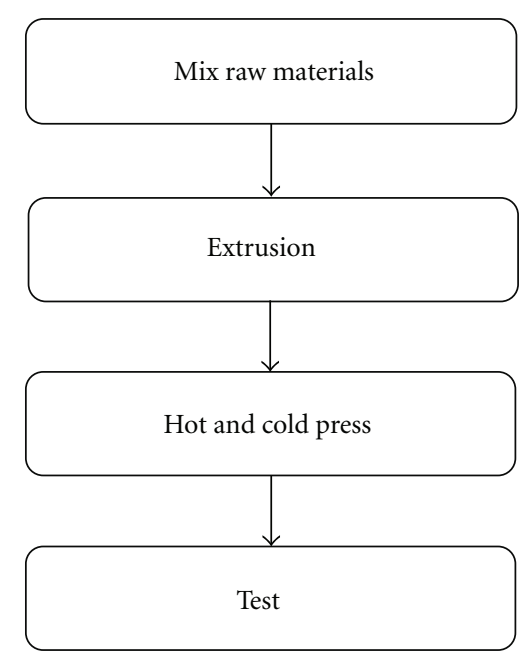

Figure 1: Flow chart of the methodology.

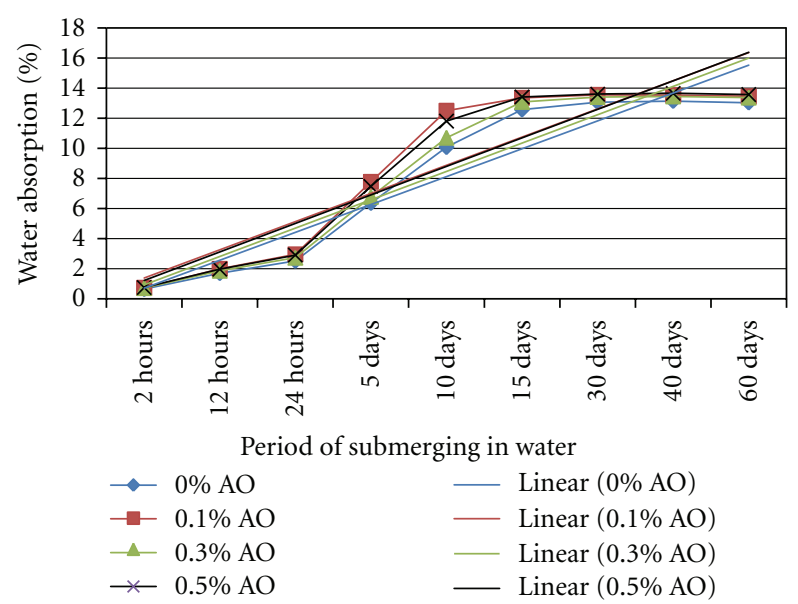

FIGURE 2: Graph water absorption of counterrotating twin-screw extruder.

\section{Results and Discussion}

The water absorption of different sample compositions as a function of period submerging in water after 60 days is presented in Figures 2 and 3. From the testing, samples produced by using counterrotating twin-screw extruder exhibits a better trend compared to corotating twin-screw extruder as shown below.

Figure 2 shows graph water absorption for counterrotating, and Figure 3 represents graph water absorption for corotating twin-screw extruder. According to Klyosov [1], water absorption for wood plastic composite (WPC) typically is $0.7-2 \%$ after $24 \mathrm{~h}, 1-5 \%$ after a week, and up to $18-22 \%$ after several months. This result could be explained by wood chemical composition itself which is because wood fiber contains high hydrophilic content (cellulose and hemicelluloses). Cellulose and hemicelluloses are mostly responsible for the high water absorption of natural fibers, since they contain numerous accessible hydroxyl groups. Besides that, poor adhesion between fiber particles and

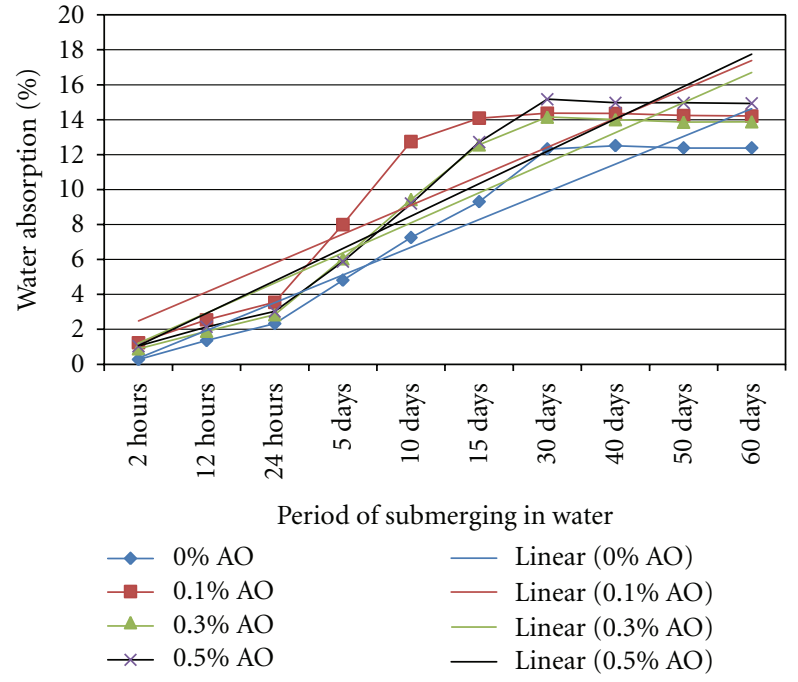

FIGURE 3: Graph water absorption of corotating twin-screw extruder.

TABLE 2: Water absorption rate of counterrotating twin-screw extruder.

\begin{tabular}{lcccc}
\hline Period of submerging & \multicolumn{4}{c}{ Water absorption (\%) } \\
in water (days) & 0.0 & 0.1 & 0.3 & 0.5 \\
\hline 2 hours & 0.65 & 0.73 & 0.72 & 0.75 \\
1 & 2.52 & 2.95 & 2.73 & 2.91 \\
15 & 12.57 & 13.36 & 13.09 & 13.42 \\
30 & 13.06 & 13.56 & 13.41 & 13.61 \\
60 & 13.03 & 13.46 & 13.37 & 13.58 \\
\hline
\end{tabular}

TABLE 3: Water absorption rate of corotating twin-crew extruder.

\begin{tabular}{lcccc}
\hline Period of submerging & \multicolumn{4}{c}{ Water absorption (\%) } \\
in water (days) & 0.0 & 0.1 & 0.3 & 0.5 \\
\hline 2 hours & 0.28 & 1.23 & 0.89 & 1.04 \\
1 & 2.33 & 3.55 & 2.84 & 3.02 \\
15 & 9.30 & 14.09 & 12.56 & 12.72 \\
30 & 12.32 & 14.38 & 14.15 & 15.17 \\
60 & 12.38 & 14.21 & 13.88 & 14.93 \\
\hline
\end{tabular}

the polymer matrix generates void spaces around the fiber particles. These lead to higher water uptake in WPC. There is no doubt that maleated polymers as coupling agents often decrease water absorption by WPC, as well as increase tensile and flexural strength of WPC. However, they often increase insignificantly the flexural and tensile modulus, contrary to that as would have been expected if strong covalent bonds are formed between the fillers (saw dust and rice husk) and the polymer matrix (rHDPE).

From Tables 2 and 3, it clearly showed water absorption rates for all samples produced by corotating twin-screw extruded higher than samples produced by counterrotating 


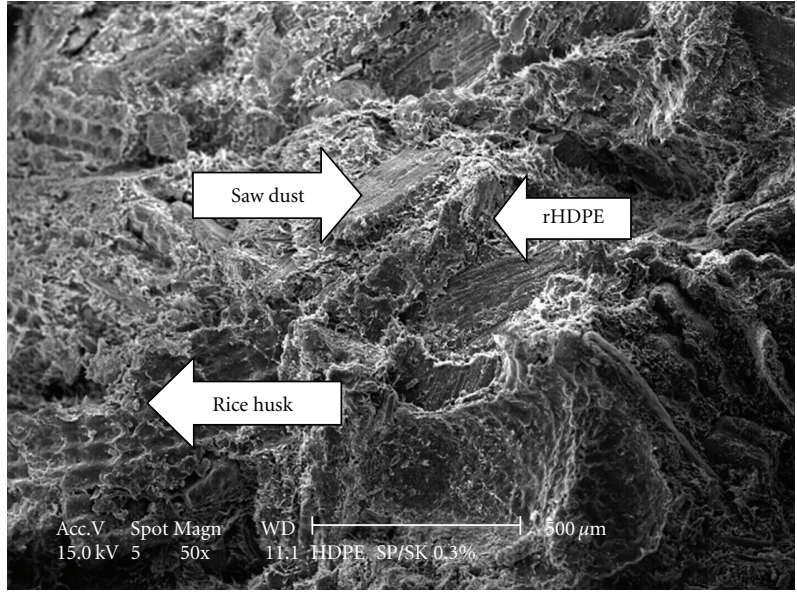

(a)

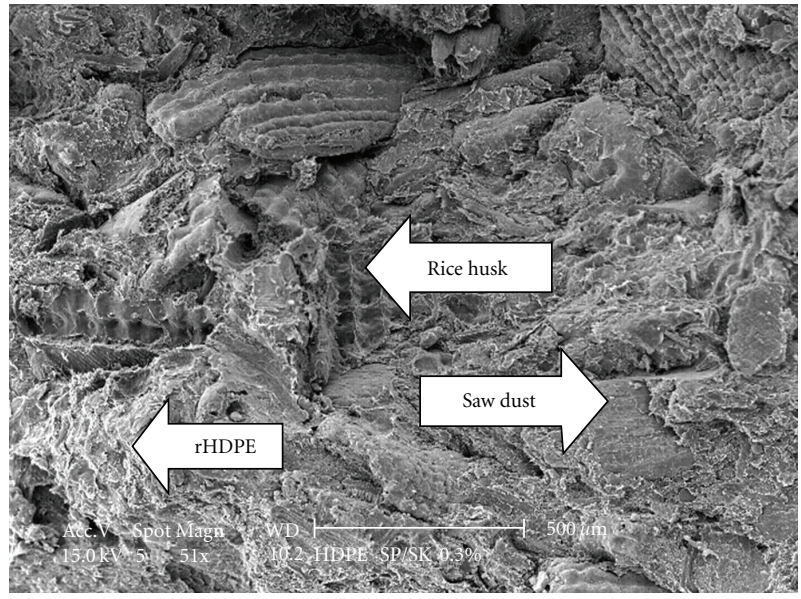

(b)

Figure 4: SEM photographs of sample produced by (a) counterrotating twin-screw extruder and (b) corotating twin-screw extruder.

twin-screw extruder. The results indicate that counterrotating twin-screw extruder has a better mixing compared to corotating twin-screw extruder.

A morphology study was carried out using scanning electron microscopy (SEM) to evaluate the fractured surface of samples. The changes in morphology are important to predict fiber interaction with the matrix in composites.

SEM photographs of fractured surfaces of samples produced by counterrotating and corotating twin-screw extruders are shown in Figures 4(a) and 4(b), respectively. Figure 4(a) shows better dispersion of fiber in the matrix compared to Figure 4(b). Better dispersion of fiber in composites leads to high performance on physical and mechanical properties.

Both photographs show a lot of rice husk content rather than saw dust; this is since high amount of saw dust had been used in the composition compared to saw dust.

\section{Conclusion}

Adding agrobased fibers to recycled high-density polyethylene produce composites that increased the water absorption rates compared to composites containing no fiber. The addition of antioxidants to these composites gives additional benefits. These antioxidants are highly effective, low volatile, and hydrolysis resistant for coating resins. They protect the resin polymer against oxidation during resin synthesis, processing (thermal curing and overbaking), and the designed life-time of the final coating. They also provide excellent protection against change of physical properties caused by excessive heat exposure that lead to high water absorption in wood plastic composites.

Types of extrusion machine also play a big role in WPC processing. The results show that counterrotating twin-screw extruder is the best method to produce WPC. This is because heat-sensitive polymers and low temperature extrusion for fibers are utilized. Furthermore, moisture removal also can be implemented during extrusion process through vacuum venting as discussed by Gardner and Murdock [9].

\section{References}

[1] A. A. Klyosov, Wood Plastic Composites, John Wiley \& Sons, New York, NY, USA, 2007.

[2] W. Wang, M. Sain, and P. A. Cooper, "Study of moisture absorption in natural fiber plastic composites," Composites Science and Technology, vol. 66, no. 3-4, pp. 379-386, 2006.

[3] A. R. Sanadi, D. F. Caulfield, and R. E. Jacobson, "Agrofiber/thermoplastic composites," Paper and Composites from Agro-Based Resources, pp. 377-401, 1997.

[4] M. P. Walcott and K. A. Englund, "A technology review of wood-plastic composites," in Proceedings of the 33rd International Particleboard/Composite Materials Symposium, pp. 103$111,1999$.

[5] P. V. Joseph, M. S. Rabello, L. H. C. Mattoso, K. Joseph, and S. Thomas, "Environmental effects on the degradation behaviour of sisal fibre reinforced polypropylene composites," Composites Science and Technology, vol. 62, no. 10-11, pp. 1357-1372, 2002.

[6] N. Stark, "Influence of moisture absorption on mechanical properties of wood flour-polypropylene composites," Journal of Thermoplastic Composite Materials, vol. 14, no. 5, pp. 421-432, 2001.

[7] H. Azman and K. Sivaneswaran, The effect rice husk ash fillers on processability of ABS impact modified PVC-U, Doctoral thesis, Department of Polymer Engineering University of Technology Malaysia, Skudai, Malaysia, 2006.

[8] S. M. Zabihzadeh, "Water uptake and flexural properties of natural filler/HDPE composites," BioResources, vol. 5, no. 1, pp. 316-323, 2010.

[9] D. J. Gardner and D. Murdock, Extrusion of Wood Plastic Composites, University of Maine, Orono, Me, USA, 2010. 

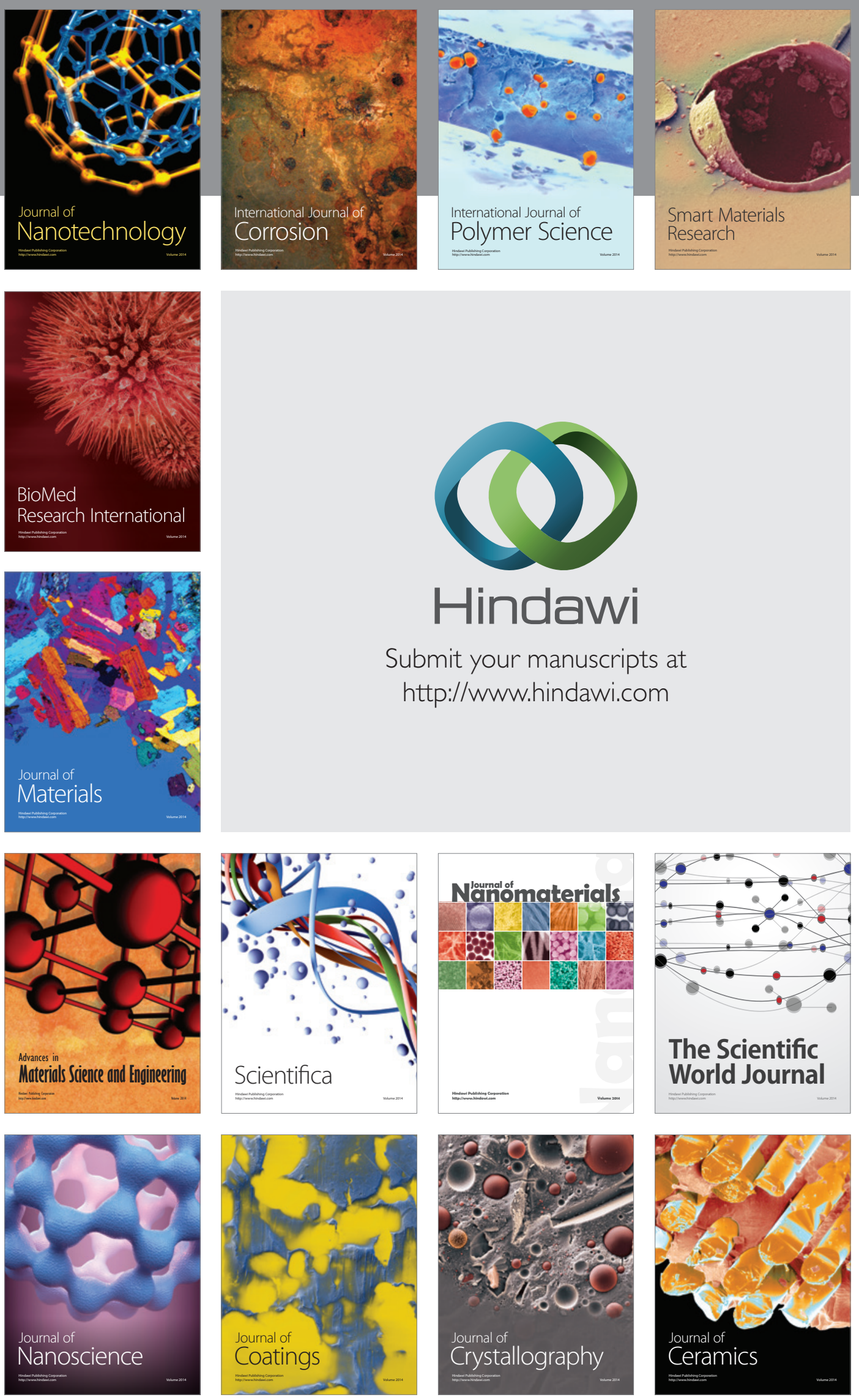

The Scientific World Journal

Submit your manuscripts at

http://www.hindawi.com

\section{World Journal}

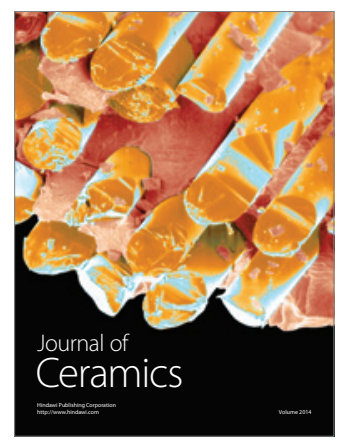

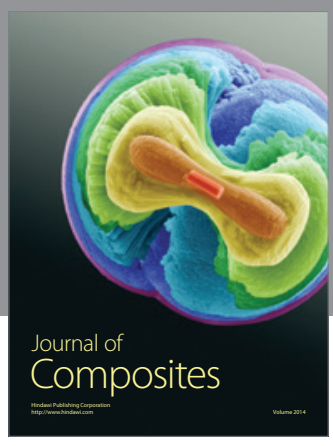
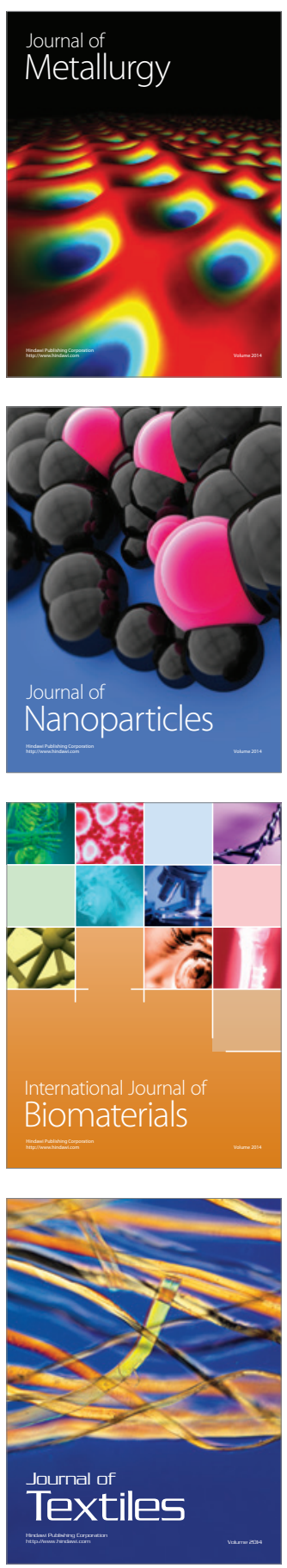